\title{
Colombia, Brasil, Argentina y Chile: práctica educativa y orientaciones pedagógicas ${ }^{1}$
}

\author{
Colombia, Brazil, Argentina and Chile: educational practice and \\ pedagogical orientations
}

Colômbia, Brasil, Argentina e Chile: estágio supervisionado e orientações pedagógicas

\author{
Carlos Federico Ayala Zuluaga ${ }^{2}$ \\ Universidad de Caldas (Colombia) \\ Roberto Tadeu Iaochite ${ }^{3}$ \\ Universidade Estadual Paulista (Brasil) \\ Samuel de Souza Neto ${ }^{4}$ \\ Universidade Estadual Paulista (Brasil)
}

Recepción: 10/11/2016

Evaluación: 13/06/2017

Aceptación: 24/08/2017

Artículo de Investigación - Reflexión

DOI: https://doi.org/10.19053/01227238.7576

\section{RESUMEN}

El presente artículo discute aspectos de la Práctica Educativa en cuatro universidades oficiales, o del estado, en: Colombia, Brasil, Argentina, y Chile y está en relación con las orientaciones pedagógicas propuestas por Feiman

1 Este artículo es producto de la tesis de doctorado titulada “Orientações Pedagógicas no Estágio Supervisionado de Educação Física na Colômbia, Brasil, Argentina e Chile”, auspiciada y financiada Universidad de Caldas, (PAEDEX/AUIP - internacionalização Doutorado - Bolsa PROPG/UNESP. Projeto: PPGCM - LP: FPCT e PPGE - LP: FPTD - Estagio Curricular Supervisionado: Cenários dos Modelos de Formação Docente.

2 Enviar correspondência. Doctor en Ciencias de la Motricidad, Profesor Asociado Universidad de Caldas, Grupo Cumanday Actividad Física y Deporte; Grupo de Pesquisa NEPEF: FPCT e do Grupo de Pesquisa DOFPPEN. Email: federico.ayala@ucaldas. edu.co.

3 Doutorado em Educação, Professor Assistente Doutor da Universidade Estadual Paulista Júlio de Mesquita Filho, Brasil, Pesquisador do NEPEF/Unesp e DOFPPEN/Unesp. Email: iaochite@rc.unesp.br.

4 ós-Doutor no Centre de recherche interuniversitaire sur la formation et profission ensignante (CRIFPE), Université de Montréal, Professor do Departamento de Educação da Universidade Estadual Paulista - UNESP-Rio Claro (RC), Grupo de Pesquisa NEPEF: FPCT (2002) e do Grupo de Pesquisa DOFPPEN. Email: samuelsn@rc.unesp.br. 
(1990) e Imbernon (2007). El enfoque de la investigación es cualitativo y busca comprender aspectos intrínsecos, regionales, culturales, y políticos de la práctica educativa relacionada con orientaciones pedagógicas, mediante un proceso de preanálisis fluctuante de la información, la codificación e identificación de categorías, y finalmente la descripción, categorización, inferencia e interpretación de la información recogida. En los resultados se evidencia que las orientaciones pedagógicas convergen en la acción, la reflexión, el conocimiento, el uso de las Tecnologías digitales de información y comunicación, y en el desarrollo de la identidad docente. Conclusionesprincipales:lasorientaciones pedagógicas predominantes en la práctica educativa oficial son la académica y la tecnicista, y en las universidades son la tradicional, académica, de eficacia social, hermenéutica y la centrada en procesos.

Palabras clave: política pública docente; formación docente; practicante-profesor; identidad docente.

\section{ABSTRACT}

This article discusses aspects of the Educational Practice in four state universities in: Colombia, Brazil, Argentina, and Chile. We also take into account the conceptual orientations in teacher training proposed by Feiman (1990) and Imbernon (2007). The research approach is qualitative and aims to understand intrinsic, regional, cultural, and politicalaspectsofeducational practice related to pedagogical orientations, through a process of fluctuating preanalysis of information, coding and identification of categories. The final stage consisted of description, categorization, inference and interpretation of the information collected. The results show that pedagogical orientations converge in action, reflection, knowledge, the use of digital information and communication technologies, and in the development of the teaching identity. Main conclusions: the predominant pedagogical orientations in the state education are the academic and the technical, and in the universities were identified the traditional, academic, social effectiveness, hermeneutics, and process-centered.

Keywords: teaching public policy; teacher training; practitioner-teacher; teaching identity.

\section{RESUMO}

O artigo discute aspectos do Estagio Supervisionado em quatro universidades oficiais ou da nação, uma por cada país, em Colômbia, Brasil, Argentina e Chile e está em relação com as Orientações Pedagógicas propostas por Feiman (1990) e Imbernon (2007). Enfoque qualitativo de pesquisa, procurando compreender aspectos intrínsecos, regionais, culturais e políticos do Estagio Supervisionado relacionado com as Orientações Pedagógicas, mediante um processo de pré-análise flutuante da informação, a codificação e identificação de categorias e finalmente a descrição, categorização, Inferência e Interpretação da informação recolhida. Resultados: as Orientações Pedagógicas convergem na ação, reflexão, ação, no conhecimento, no uso da Tecnologia Digital da Informação e Comunicação, e no desenvolvimento da identidade docente; conclusões principais: As Orientações Pedagógicas predominantesnoEstagioSupervisionado são Académica e Técnicista no oficial, nas universidades são Tradicional, Académica, Eficácia Social, Hermenêutica e Centrada nos Processos.

Palavras chave: política pública docente; formação docente; educação física; estagiárioprofessor.

Contextualización: Idioma Maya Sakapulteko

Contextualizadora: Delfina Arcón Puzul Ministerio de Educación de Guatemala 
TUNB'AAL TZ'IIJ:

E jun wuuj ri ki ch'aw purwe' ri b'anb'aal tujneem chapam kijab' namaq taq tub'aal aj choq'on li tinimiit, re: Colombia, Brasil, Argentina, saqara' Chile, kikan ri pixb'aneem purwe' tujneem kirya' Feiman (1990) richb'iil Imbernon (2007). E ri b'eey jun jyneem ri kiril ri b'anb'aal ki k'aslemaal saqara' kirjiij ri ch'abik riij chetaq junam rib'anik ramaal jun jupuq tinimiit ri, kib'anb'aal purwe' tujneem chapam jun kyaj pixb'aneem re ri b'anik tujneem, rmaal jun b'anb'aal re ri chomxiik riij li tzujneem, xaqara' re karaq taj riij ru utzilaal, xaqara' ki k'istaj kan otz laj rib'ixik, ru utzilaal, xaqara' ri chomxik riij li chomneem raqtajek. Chapam li chomneem ri kiltaj chapam che li b'ananeem re tujneem kikiraqkib' chapam li chaak, chapam li chomb'aal, chapam ri kojik ch'ich' re jyb'aal chomneem xaqara' tzujneem, xaqara' kiltaj chapaam ri b'antajiik li tujneel. Namaq taq k'isb'aneem: Jun kyaj pixb'aneem purwe' tujneem chapam tujb'aal re tinmiit, el li ka k'amb'ik chapam pi tujb'aal, taq tanaj, xaqara' chapam li namaq taq tujb'aal kab'an chapam ri b'anab'aal re ajar taq tziij, pa tanaj xaqara', re utzilaal re winaqiil, xaqara' kirb'aj ri b'anab'aa'l.

Retaal tz'iij: Chak pur we' li Tujneel, Tujneem re Tujneel, B'ananeem-Tujneel, $R u$ $b^{\prime}$ antajiik re Tujneel.

\section{INTRODUCCIÓN}

A finales del siglo XX y principios del XXI, los procesos educativos en varios países del mundo como EE.UU, Francia, Inglaterra, Brasil, Chile y Colombia entre otros, asumieron el reto de reformas en el campo de la educación, la pedagogía y la didáctica, que fueron influenciadas por aspectos socio-económicos "que por su vez expresan una concepción de educación orgánica al modelo económico en el proceso globalizado de acumulación flexible ${ }^{\prime \prime 5}$, centrándose en la formación humana y ciudadana de los sujetos, a partir de los procesos de la enseñanza y el aprendizaje, lo que por ende influyó en la formación de profesores.

También los aspectos económicos y de la tecnología fueron la base para el fomento del conocimiento y la ciencia en el siglo anterior, procurando el crecimiento y desarrollo de los países y en convenio o parecería con las aperturas económicas ${ }^{6}$, el acceso a la producción masiva y los cambios en las normas laborales 7 , ya nivel científico se presenta un predominio de las ciencias positivistas en el fomento del conocimiento y bajo el auspicio del Neoliberalismo con su postulado de "sobrevivir" mediante el desarrollo de competencias.

Lo anterior tuvo una fuerte incidencia en lo político y social, en el desarrollo de los procesos y sistemas educativos orgánicos con dinámicas culturales y

5 Eliza Bartolozzi Ferreira. Ensino Meio no Brasil: Os desafios das políticas de garantia do direito a sua universalização. Linhas Críticas, Revista da Faculdade de Educação - UnB. Brasília, (2011). 17 (34): 507-525.

6 Claudio Rama. La conformación diferenciada de un nuevo subsistema tecnológico universitario en América Latina. Revista de la Educación Superior. Vol. XLIV (1); No. 173 (2015): 11-46.

7 Estela Ruiz Larraguivel. Los técnicos superiores universitarios. Diferenciación educativa, estratificación social y segmentación del trabajo. Revista Mexicana de Sociología, Vol. 71, No. 3, (2009): 557-584. 
sociales muy dinámicas ${ }^{8}$, presentándose relaciones entre saberes y conocimientos teórico-prácticos orientados a formar profesores.

Perrotta ${ }^{9}$, de frente a estas nuevas realidades manifiesta que en los procesos de educación y formación se centralizan en fortalecer las competencias, como respuesta a requerimientos y exigencias neoliberales económicas, laborales, globalizadas e incluso tecnológicas, que son regularizadas por mercados y acciones políticas capitalistas y en detrimento de los procesos que deben corresponder a las regiones.

En América Latina estos postulados de desarrollo, dentro del marco de cambios y reformas educativas, son cuestionados por la fuerte influencia de los principios ideológicos de mercado del Neoliberalismo, de la Globalización y del Capitalismo ${ }^{10}$, lo que ha generado luchas y manifestaciones de rechazo en contra de estos mismos postulados, los cuales no permiten llevar a cabo adecuados procedimientos de gestión, financiamiento y evaluación de los sistemas educativos.

Presentándose, en torno a los desarrollos educativos ineficiencias administrativas de los recursos, generando tensiones y desencuentros para regular el desarrollo de las comunidades y sociedades en lo local y lo global desde lo formativo, los cuales se administran con medidas políticas, económicas y administrativas dejando de lado los componentes educativos ${ }^{11}$.

Por lo que Freitas ${ }^{12}$ plantea que la formación de profesores debe dar respuesta a los requerimientos sociales, educativos, laborales y políticos en contraposición a las exigencias del Neoliberalismo y la Globalización, mediante la articulación de procesos de enseñanza y aprendizaje, de investigación y extensión, o sea en una relación articulada entre la escuela y la universidad, mediante la organización y desarrollo de proyectos en torno y con las comunidades, en una reconstrucción de relaciones para una nueva escuela profesionalizante, la valoración del ejercicio docente profesional, profesionalizante y de profesión, esto como "condición para una educación emancipadora de las nuevas generaciones y de formación de educadores intelectuales de un nuevo tipo, para la formación de todos los profesores como pedagogos comprometidos con la actualidad"13.

Por eso, se debe entender que la formación y los conocimientos pedagógicos que poseen los profesores y alumnos, los hace actores y figuras centrales de los procesos educativos y los directamente involucrados, pues desde sus experiencias, percepciones, ideas y representaciones de las realidades y de los contextos educativos; conocimientos, saberes y vivencias es que se estructuran las

\footnotetext{
8 Ibídem Rama (2015).

9 Daniela Perrotta. Organismos Internacionales y Políticas en Educación Superior: su aporte al campo de estudios de la educación superior y a la construcción de políticas públicas. Revista de la Educación Superior. Vol. XLIV (1); No. 173. (2015): 181-188.

10 Carlos Bauer y Luis Paiva. O Sindicalismo docente universitário da Argentina, Brasil, Colômbia, México e sua resistência à contrarreforma universitária. Rev. hist.edu.latinoam - Vol. 19 No. 28, enero - junio 2017 - ISSN: 0122-7238 - pp. 109 - 128.

11 Dalila Andrade Oliveira, "Reformas educativas y redefinición de las fronteras entre lo público y lo privado", en Políticas de privatización, espacio público y educación en América Latina, eds. Pablo Gentili, Gaudêncio Frigotto, Roberto Leher y Florencia Stubrin. (Santa Fe: Homo Sapiens Ediciones, 2009), 121-134.

12 Carmen Lucia Freitas de Castro, "Novas Políticas de Formação: Da concepção negada à concepção consentida”, en Trajetórias e perspectivas da formação de educadores, ed. Raquel Lazzari Leite Barbosa. (São Paulo: Editora UNESP, 2004), 89-116.

13 Ibídem.
} 
Orientaciones Pedagógicas (OP), que luego en el ejercicio estudiantil y docente son las que intervienen directa o indirectamente en los procesos formativos de profesores.

Condicionalmente, las "Orientaciones Pedagógicas" - OP en adelante - contribuyen y permiten construir y reconstruir el conocimiento hacia un entendimiento de lo que es enseñar, aprender, educar y ejercer prácticas docentes y educativas ${ }^{14}$, con base en la epistemología de lo teórico y lo práctico en general, luego que estas se presentan en la historia de vida educativa de los actores ${ }^{15}$, por lo que "las orientaciones conceptuales corresponderán, de una forma u otra, a los criterios de epistemología, ontología y metodología que de los procesos o programas de investigación en educación y del concepto de sociedad, su educación y sus acciones"16.

El mismo Imbernón (2007) nos dice que para comprender las OP se deben analizar como práctica social no neutra que incide en los sujetos inseridos, con las concepciones y filosofías epistemológicas correspondientes de lo que es formación y educación, lo que es profesionalización, profesionalidad y profesión, o sea, que los profesores y estudiantes se apropien de los saberes (conocimientos y experiencias) para fundamentar los enfoques que se tienen frente a los procesos de enseñar y de aprender, desde la apropiación de la crítica, la independencia, el análisis, la innovación y la participación comprometida en procesos educativos, comunitarios, sociales, investigativos, realistas y contextuales.

Por ende, las OP las clasificamos, en este artículo, como Conservadoras y Emergentes. Presentamos en las OP Conservadoras la Tradicional que tiene como base esencial la apropiación del aprendizaje teórico; la OP Tecnicista que se fundamenta en formar para la eficacia laboral y productiva social, y la OP Academicista que procura por fortalecimiento académico e investigativo. Las OP Emergentes son la Naturalista basada en la Escuela Nueva; la Centrada en los Procesos que busca la construcción del conocimiento, a partir de las experiencias vividas de los sujetos inmersos y comprometidos con los procesos, y la Hermenéutica que se centra en el fomento de la reflexión, el análisis y la crítica a los procesos.

Lo que nos permite entender que las OP para la formación de profesores, se fundamentan en objetivos y enfoques educativos del enseñar y del aprender, desde visiones y postulados meramente educativos y formativos de intercambio recíproco entre profesores y estudiantes - objeto (la enseñanza) y el sujeto (el aprendizaje) - no los ideológicos y político-económicos de los sistema educativos.

En torno al otro concepto abordado en este artículo que es la Práctica Educativa (para unificar se reconocerá como PE), tenemos que la PE en la Universidad de Caldas (Colombia) se denomina Práctica Profesional Docente (PPD); en la Universidad Estadual Paulista (Brasil) es Estágio Supervisionado (ES); Universidad Nacional de La Plata (Argentina) titulada como Observación

14 Sharon Feiman-Nemser, "Teacher Preparation: Structural and conceptual alternatives", en Handbook of research on teacher education ed. Robert Houston. (New York: Mac Millan, 1990), 212-233.

15 Hermila Loya Chávez, "Los Modelos Pedagógicos en la Formación de Profesores", Revista Ibero-americana de Educación. Organización de Estados Ibero-americanos para la Educación, la Ciencia y la Cultura (OEI). (2008): sp.

16 Francisco Imbernón, La Formación y el Desarrollo Profesional del Profesorado: Hacia una nueva cultura profesional (Barcelona: Editorial Grau, 2007), 35. 
y Prácticas de Enseñanza en Educación Física, y Práctica Profesional Final en la Universidad Metropolitana de Ciencias de la Educación (Chile).

La Universidad y la Escuela en estos tiempos se han acercado mucho en el desarrollo de procesos educativos a partir la PE, ya que esta como objeto de investigación presenta igual sentido y significado en las universidades objeto de estudio, siendo reconocida como espacio de formación, donde se afirman y reestructuran conceptos, se sistematizan conocimientos, saberes y experiencias y se interpretan realidades en la coexistencia del día a día educativo, desde la comprensión de un espacio de "actividad práctica, pero teórica, instrumentalizada de la praxis docente, entendida esta como actividad teórica de conocimiento, fundamentación, diálogo e intervención, esta, sí, objeto de la praxis" ${ }^{17}$ y de encuentro comprensivo en la Formación de Profesores, específicamente de Educación Física, durante el ejercicio docente que se lleva a cabo entre Universidad y Escuela.

Pues la PE, es espacio educativo y formativo de tiempo y de permanencia constante - del practicante-profesor - en la escuela o en una comunidad educativa, durante su mismo proceso de formación ${ }^{18}$, en el cual adquiere nuevos aprendizajes desde conocimientos, experiencias y saberes, consciencia política y social, habilitación y perfeccionamiento para el ejercicio docente profesional, ${ }^{19}$ mediante la aproximación a las realidades y actividades teóricas pedagógicas en las cuales actúa, ligando la teoría y la práctica ${ }^{20}$ (Ayala, Franco y Ayala, 2015; Melo, 2008; Milanesi, 2008).

Por eso se debe observar la PE como proceso que fomenta la apropiación de los conocimientos adquiridos durante la formación y el aprendizaje en el pregrado ${ }^{21}$ y colocados en práctica en el contexto escolar, por el mismo practicante-profesor, basados en la responsabilidad, el compromiso, la planificación, el control y la valoración del(s) proceso(s) ${ }^{22}$ (Ayala et al., 2015; Faría, Corrêa y Bressane, 1982).

Ayala et al. (2015), Rama (2015) y Freidson (1998) ${ }^{23}$, plantean que mediante la integración de los componentes de aprendizaje y de enseñanza práctica, en torno al conocimiento y apropiación de las OP y educativas (pedagogía, didáctica, experiencias y disciplinar), en la PE se podrán desarrollar competencias formativas y educativas que resignifiquen y forjen nuevos conocimientos, saberes, aprendizajes y enseñanzas.

Por tanto Carvalho $(1985)^{24}$, nos da a entender que en los procesos de formación de profesores en estos contextos de la investigación, la PE tiene relevancia en las

17 Maria Socorro Lucena Lima y Selma Garrido Pimenta, Estágio e Docência (São Paulo: Cortez Editora, 2004$), 45$.

18 Geovana Ferreira Melo, "Estágio na Formação Inicial de Professores: Aguçando o olhar, desenvolvendo a escuta sensível”, en Estágio Supervisionado e Prática de Ensino: Desafios e possibilidades, eds. Lázara Cristina da Silva y Maria Irene Miranda (Araraquara: Junqueira\&Marin Editores, 2008), 85-113.

19 IrtonMilanesi, O estágio interdisciplinar no processo de formação docente (Cáceres: UNEMAT Editora, 2008).

20 Carlos Federico Ayala Zuluaga, Alejandra María Franco Jiménez y José Enver Ayala Zuluaga, "Prácticas profesionales educativas (PPE): reflexión desde la educación física", Revista Latinoamericana de Ciencias Sociales, Niñez y Juventud, 13, No. 2 (2015) 595-607. DOI: http://dx.doi.org/10.11600/1692715x.1323100713.

21 Ibídem.

22 Alfredo Gomes de Faria Junior, Eugénio da Silva Corrêa y Riselaine da Silva Bressane, Prática de ensino em educação física: estágio supervisionado (Rio de Janeiro: Interamericana, 1982).

23 Eliot Freidson, Renascimento do profissionalismo: teoria, profecia e política (São Paulo: Editora da Universidade de São Paulo, 1998).

24 Anna Maria Pessoa de Carvalho, Prática de Ensino: os estágios na formação do professor (São Paulo: Biblioteca Pioneira de Ciências Sociais, 1985). 
licenciaturas de Educación Física, pues la aproximación a las realidades escolares y educativas les permitirá a los practicantes-profesores, aprender y solidificar los componentes de programación, planificación y valoración del ejercicio docente, a partir de lo aprendido y enseñado, ya sea analizando, confrontando, discutiendo, organizando, sistematizando, integrando, aplicando y evaluando sus saberes, experiencias y realidades de enseñanza.

Por todo lo anteriormente expuesto, podemos hacer relaciones que nos permitirán comprender cómo desde los aspectos legislativos y normativos se desarrollan PE en Educación Física, desde la perspectiva de las Orientaciones Pedagógicas (OP), y haciendo recorte en los países mencionados (Colombia, Brasil, Argentina y Chile), teniendo en cuenta sus desarrollos históricos, económicos, sociales y políticos en torno a los aspectos que conciernen a la educación, específicamente, las relaciones entre universidad y escuela en la acción y las concepciones educativas y pedagógicas, los procesos legislativos y normativos reguladores de las PE y del actuar de los practicantes-profesores y los Proyectos Políticos Pedagógicos de cada programa de formación.

\section{Escuela - Universidad y la Práctica Educativa (PE)}

Inicialmente se asumen las normas nacionales, departamentales (estadual), municipales y universitarias, que permiten el desarrollo la PE en la relación Universidad y Escuela, las relaciones académicas de los profesores escolares, los asesores universitarios y los practicantes-profesores. Posteriormente se exhiben las normas de la PE en relación con las OP y los actores de los procesos. Se pretende exponer los encuentros y desencuentros normativos de la PE en cada país, y así regular y develar las OP que determinan la construcción, direccionamiento, administración y lo educativo, de la formación de maestros en la región.

En la descripción que se presenta sobre el desarrollo de la Práctica Educativa (PE), a partir de los aspectos legislativos, normativos y de la estructura académica curricular de la misma PE, se buscó comprender lo que hay detrás de estos procesos, lo que subyace en ellos y sus relaciones en la educación del nivel básico y superior, en donde pueden mediar convenios y/o acuerdos entre movimientos o grupos con intereses u objetivos comunes y así defenderlos y fortalecerlos de manera mancomunada. ${ }^{25}$

\section{Universidad de Caldas - UdeC - Colombia}

Este ítem se estructuró, con base en las directrices normativas en las esferas de la Nación, de la Universidad, de las diferentes regulaciones de la Facultad y de la coordinación de la PE. en la Universidad de Caldas, los procesos de la PE se desenvuelven en las legislaciones, reglamentos, conocimientos y aspectos

25 Daniela Perrotta. Organismos Internacionales y Políticas en Educación Superior: su aporte al campo de estudios de la educación superior y a la construcción de políticas públicas. Revista de la Educación Superior. Vol. XLIV (1); No. 173. (2015): 181-188. 
históricos, teniendo una visión y fundamentación holística: o sea el porqué, el para qué y el cómo se lleva a cabo en la institución.

La PE, en esta Universidad se denomina "Práctica Profesional Docente (PPD)" para todos los cursos de licenciatura de la institución. Así que es aceptada y asumida por el programa de Licenciatura en Educación Física, Recreación y Deporte de la Facultad de Ciencias para la Salud. Sin embargo, el componente pedagógico, didáctico, curricular y metodológico es orientado por el departamento de Estudios Educativos de la Facultad de Artes y Humanidades, y tiene su fundamento de formación en la idealización del "Decreto 272 de 1998 que instala la Pedagogía como disciplina fundante del currículo para la formación de educadores" 26.

Con base en la fundamentación anterior, se busca en los programas orientar los procesos de formación para el desarrollo de actividades de enseñanza y aprendizaje, sustentadas en lo epistemológico, en los procedimientos y en los saberes disciplinares y pedagógicos, articulando niveles, ciclos y procesos educativos, formativos, pedagógicos y didácticos en el contexto que se desenvuelve el alumno ${ }^{27}$. Por eso se entiende que la PE en la Universidad de Caldas tenga sus bases y fundamentos de desarrollo en los aspectos jurídicos y legislativos, esencialmente en la Ley General de Educación 30/1992 (Educación Superior) apoyada en la Ley 115 de 1994, del Sistema Educativo Colombiano, con las correspondientes resoluciones y decretos que la reglamentan. Se tiene también el Decreto 2566/2003, que es el responsable por las condiciones mínimas de calidad y requisitos para el ofrecimiento y desenvolvimiento de programas académicos de la educación superior. Además se presenta la Resolución 1036/2004, en la cual se definen las características específicas de calidad para los programas y especializaciones en Educación.

Sustentado en las normas y planos mencionados anteriormente, pero específicamente los relacionados con las finalidades y los objetivos de los procesos de formación de profesores en Colombia y en la Universidad de Caldas, en el artículo 109 de la Ley 115/1994 ${ }^{28}$, se exhiben las directrices respectivas, para formar un docente que sea investigador de y en la educación, con idoneidad pedagógica y didáctica y que su ejercicio docente se relacione con el contexto de la Escuela.

En el ámbito legislativo de Colombia, según la Resolución 5443/2010, se definen las características específicas de calidad de los programas de formación profesional en la educación; en el artículo seis, se determina la importancia de la PE en la enseñanza, en el aprendizaje y en el desarrollo de las competencias educativas. Por eso, se presentan las directrices que orientan y contribuyen en el desenvolvimiento de esta en los espacios formativos o en las instituciones escolares.

Lo que se propone en esta resolución es que el campo de la PE sea un espacio dondeel practicante-profesor desenvuelva actividades de enseñanza y aprendizaje, donde articule los diferentes conocimientos, conceptos y procedimientos

26 "Reglamento de Práctica e Investigación Educativa de los Programas de Licenciatura de la Universidad de Caldas". Facultad de Artes y Humanidades, Departamento de Estudios Educativos (Colombia, 2002). Universidad de Caldas (UdeC).

27 "Resolución 5443 de 2010. Promulgada junio 30 de 2010". (Colombia, 2010). Ministerio de Educación Nacional (MEN), www. mineducacion.gov.co/.../articles-243532_archivo_pdf_res6966.pdf.

28 "Ley General de Educación. Ley 115 de 1994. Promulgada febrero 08 de 1994". (Colombia, 1994). http://www.mineducacion.gov. co/1621/articles-85906_archivo_pdf.pdf. 
adquiridos con los saberes disciplinares, didácticos, epistemológicos, experienciales y pedagógicos.

En la Universidad de Caldas, además de la reglamentación nacional, las autoridades respectivas proponen para el desenvolvimiento de la PE acuerdos y resoluciones, como las Políticas Curriculares, el Plan de Desarrollo y el Reglamento Estudiantil de la Institución, los cuales determinan las directrices y caminos a adoptar en la PE. Estos documentos contribuyen en la planeación, regularización y estructuración de estrategias de seguimiento, acompañamiento, mejoramiento, investigación, evaluación, reflexión y crítica en torno del desarrollo de competencias profesionales y docentes del practicante-profesor durante el proceso de la $\mathrm{PE}^{29}$.

Se comprende que el desarrollo de la PE en la Universidad de Caldas se fundamenta esencialmente en las directrices y principios políticos del orden internacional y nacional, que llevan en cuenta propuestas y políticas educativas con relación a los aspectos que buscan renovaciones en los sistemas educativos en general, con el pretexto de mejorar la educación y la formación de los profesionales de la docencia.

En el Proyecto Político Pedagógico (PPP) del programa de Licenciatura en Educación Básica, con énfasis en Educación Física, Recreación y Deporte, la PE tiene como objetivo central desenvolver en los licenciandos la capacidad de involucrarse e intervenir científica, humana y pedagógicamente en las instituciones y en las comunidades educativas, mediante el ejercicio docente.

Por tanto, se entiende que la formación de los alumnos se desenvuelve en torno a lo que se denomina "Modelo Cognitivo-Experiencial-Reflexivo", en el cual se busca indagar y comprender los actos educativos, pedagógicos y didácticos en la formación y específicamente en la PE, a través del fomentar la participación activa, la crítica fundamentada, la reflexión constante, los diálogos académicos y el debate responsable, con base en la vivencia académica, experimentación científica y demostración del ejercicio docente.

En cara a esta caracterización, se observa en el perfil profesional la perspectiva de formar un profesor que tenga como identidad en el aprendizaje de la docencia, la orientación para apropiarse de los conocimientos adquiridos, confrontarlos con las realidades educativas y pedagógicas mediante la reflexión y la crítica constructiva.

\section{Universidade Estadual Paulista - UNESP - Brasil}

En Brasil el proceso de la Práctica Educativa se denomina "Estágio Curricular Supervisionado (ECS)" e inicia su consolidación con la Ley $\mathrm{n}^{\mathrm{o}}$ 6.494/197730, que dispone sobre las prácticas de estudiantes en establecimientos de enseñanza superior y profesionalizante del $2^{\underline{0}}$ Grado y el suplementario

29 "Política Curricular. Programa de Licenciatura en Educación Básica con Énfasis en Educación Física, Recreación y Deporte" (Colombia, 2010) Consejo Académico Universidad de Caldas (UdeC): 14.

30 "Lei No 6.494, de 7 de dezembro de 1977. Dispõe sobre o estágio de estudante de estabelecimentos de ensino superior e de $2^{\circ}$ Grau Regular e Supletivo, nos limites que especifica e dá outras providências" (Brasil, 1977). Casa Civil. Subchefia para Assuntos Jurídicos. http://www.planalto.gov.br/ccivil_03/leis/L6494.htm. 
y de otras providencias sobre quien participa de la práctica, las condiciones, el tiempo y los objetivos de este. El Decreto-Ley $n^{0} 8.859 / 1994^{31}$, que modifica algunos dispositivos de la Ley anterior (Ley $n^{-}$6.494), extiende a los alumnos de enseñanza especial, el derecho a la participación en actividades de la PPE en los contextos públicos y particulares, con el fin de proporcionar experiencias prácticas en la línea de formación del practicante-profesor.

En el Decreto $n^{0} 87.497 / 1982^{32}$, se reglamenta la Ley no 6.494 , específicamente en la definición del objetivo de la PPE, quienes son los actores, la carga horaria, la duración de esta y de la jornada, el Plazo de Compromiso del practicanteprofesor y de la institución donde se llevará a cabo la PE.

En la opinión del Consejo Nacional de Educación (CNE) CNE/CP 21/200133 del Ministerio de Educación y del Consejo Nacional de Educación, se presentan las nuevas Directrices Curriculares Nacionales para la Formación de Profesores en la Educación Básica y en el nivel superior de los programas de licenciatura de formación plena (Licenciatura y Bacharelado), determinándose el papel de los profesores en los procesos educativos nacionales y una base común para mejorar los modelos educativos existentes y, por lo tanto las características de la PE.

En el documento antes mencionado, la Resolución CNE/CP 1/2002 ${ }^{34}$, se propone articular principalmente la teoría y la práctica con base en el saber hacer y en el comprender lo que se hace, mediante procesos sistemáticos de reflexiones colectivas e individuales, en que la PE sea articulada en toda la formación docente, en lo posible desde el primer año de formación y en convenio con las escuelas, donde "[...] la práctica educativa no puede quedar sobre la responsabilidad de un único profesor de la escuela de formación, pero envuelve necesariamente una actuación colectiva de los formadores" 35 .

Las opiniones del CNE/CP 27 y 28 de $2001^{36}$, disponen el tiempo de inicio de la $\mathrm{PE}$, el lugar de desenvolvimiento, los actores involucrados, la relación con el Proyecto Político Pedagógico y la relación Universidad-Escuela.

El CNE en la Resolución 2/2002 ${ }^{37}$, artículo 3, orienta los propósitos o principios que guiarán la formación de profesores para el ejercicio profesional, teniendo coherencia entre la formación y la práctica docente, por lo que se propuso la

31 "Lei no8.859. Março 23 de 1994. D. O. U de 24/03/94. Estagiários - Aceitação pelas pessoas jurídicas de Direito Privado - Órgãos da Administração Pública e Instituições de Ensino - Requisitos - Critérios - Lei No. 6.494/77 Alteração" (Brasil,1994). Congresso Nacional. http://www4.pr.gov.br/gee/documentos/legislacao/LEI-8859-94-ESTAGIO.pdf.

32 “Decreto no 87.497. Agosto 18 de 1982. Regulamenta a Lei no 6.494, de 07 de dezembro de 1977, Regulamenta a Lei no 6.494 , de 07 de dezembro de 1977" (Brasil, 1982). Presidência da República. http://www.planalto.gov.br/ccivil_03/decreto/d87497.htm.

33 "Parecer N.o: CNE/CP 27/2001. Dá nova redação ao item 3.6, a línea c, do Parecer CNE/CP 9/2001, que dispõe sobre as Diretrizes Curriculares Nacionais para a Formação de Professores da Educação Básica, em nível superior, curso de licenciatura, de graduação plena" (2001a). (Brasil, 2001ª). Consejo Nacional de Educación (CNE). http://portal.mec.gov.br/cne/arquivos/pdf/027.pdf.

34 "Resolução CNE/CP 1/2002. Institui Diretrizes Curriculares Nacionais para a Formação de Professores da Educação Básica, em nível superior, curso de licenciatura, de graduação plena" (Brasil, 2002). (CNE). http://portal.mec.gov.br/cne/arquivos/pdf/rcp01_02.pdf.

35 Ibídem: 58 .

36 "Parecer N.o: CNE/CP 28/2001. Dá nova redação ao Parecer CNE/CP 21/2001, que estabelece a duração e a carga horária dos cursos de Formação de Professores da Educação Básica, em nível superior, curso de licenciatura, de graduação plena" (Brasil, 2001b). (CNE). http://portal.mec.gov.br/cne/arquivos/pdf/028.pdf.

37 "Resolução CNE/CP 2, de 19 de fevereiro de 2002. Institui a duração e a carga horária dos cursos de licenciatura, de graduação plena, de formação de professores da Educação Básica em nível superior” D. O. U. (Brasília DF, 2002). Ministério da Educação. 
distribución de la carga horaria, para la PE. La Resolución 7/2004 ${ }^{38}$, instituyó las Directrices Curriculares Nacionales para los programas de formación en Educación Física, en el nivel superior de graduación plena. En su artículo 5, determina y define que el proceso de la PE contribuye para consolidar y vivenciar las competencias desarrolladas en espacios académicos diferentes y con la orientación y asesoría de un profesional habilitado.

Finalmente, se decreta la Ley $\mathrm{n}^{\circ} 11.788 / 2008^{39}$, en que se reglamenta la PE en plenitud. En esta se proponen las directrices sobre la PPE para estudiantes en su definición, clasificación, las relaciones de la práctica, de la institución de enseñanza, de la parte cedente, del practicante-profesor, de la fiscalización y de las disposiciones generales. Más allá de eso, se tiene el Parecer CNE/CES № 416/2012 ${ }^{40}$, en el cual se aprueba la realización de la PE fuera del país.

La PE en la Universidad Estadual Paulista (UNESP) tiene su basamento en el artículo 1 de la Resolución UNESP 36/1996 ${ }^{41}$, en este se determinan los objetivos de la formación, la PE y la cualificación para la profesión mediante la Práctica Obligatoria.

El Consejo del Programa de Graduación en Educación Física (CCGEF, siglas en portugués) presentó una propuesta de reestructuración curricular ${ }^{42}$, en la cual se admitía la nueva estructura propuesta por el CNE, con los objetivos y las condiciones que determinaron para el desarrollo de la PPE en programas que forman maestros y que se desempeñarán en el ámbito escolar y no escolar (bacharelado) o espacio no formal de educación - clubes, centros de actividad física, escuelas deportivas, entre otros - fundamentados en las directrices curriculares resumidas anteriormente.

\section{Universidad Nacional de La Plata - UNLP -, Argentina}

En el Programa de Profesorado de Educación Física en la Universidad Nacional de La Plata, la PE es denominada como “Observación y Prácticas de Enseñanza en Educación Física I y II". En este programa se da cuenta de las políticas, de los medios, de las tendencias e innovaciones que se presentan, proponen y se asumen en el sistema de la Política Pública Educativa de la Argentina, especialmente los procesos legislativos y reglamentarios, a partir de la Reforma Educativa de 1990

38 "Resolução No. 7, Março 31 de 2004. Institui as Diretrizes Curriculares Nacionais para os cursos de graduação em Educação Física, em nível superior de graduação plena” (Brasil,2004). CNE. Câmara de Educação Superior. http://portal.mec.gov.br/cne/arquivos/ pdf/ces0704edfisica.pdf.

39 "Lei n. 11.788 setembro 25 de 2008. Disposições sobre Estágio de Estudantes" (Brasil, 2008). Presidência da República. http://www. planalto.gov.br/ccivil_03/_ato2007-2010/2008/lei/111788.htm.

40 "Parecer CNE/CES No: 416/2012: Consulta sobre estágio no exterior" (Brasil, 2012). CNE. http://www.prograd.ufu.br/legislacoes/ parecer-cneces-no-4162012-aprovado-em-8-de-novembro-de-2012-estagio-no-exterior.

41 "Resolução 36 de 7 de agosto de 1996. Obrigatoriedade do Estágio Curricular Obrigatório" (Reitoria, 1996). Universidade Estadual Paulista (UNESP).

42 "Proposta de reestruturação curricular dos cursos de licenciatura e graduação (bacharelado) em educação física do instituto de biociências da UNESP/RC" Rio Claro (2005). Conselho de cursos de graduação em educação física - CCGEF Comissão de estudo curricular - CEC. UNESP. 
y, así mismo, permite que en ese cuerpo normativo nacional sea orientada la formación docente para el ejercicio de la profesión docente.

Por eso, a partir de la Resolución $32 / 1993^{43}$ del Consejo Federal de Educación, se propone que las prácticas didácticas docentes y el sistema educativo como ejes curriculares valoricen la PE, donde son tenidas en cuenta las problemáticas contextuales, la integración de las teorías y los conocimientos disciplinares, para formar y educar profesores críticos, autónomos, creativos y comprometidos con las comunidades educativas, asimismo definió los Contenidos Básicos Comunes, implementó un sistema de evaluación de la calidad educativa y otorgó el papel de subsidiar la educación por parte del estado ${ }^{44}$.

Además se proponen directrices sobre la organización, las funciones, las características, los criterios, los parámetros y los campos de la formación educativa ${ }^{45}$; la reorganización del sistema de formación profesional de la docencia en relación con la carga horaria, la organización curricular, la acreditación institucional y la educación polimodal ${ }^{46}$; también se tienen, los principios y los criterios para el desempeño docente en la educación de jóvenes y adultos ${ }^{47}$.

Encuéntrese en la Ley de Educación Superior 24.521/1995 ${ }^{48}$, el direccionamiento de las funciones básicas de las instituciones universitarias y se busca que la Universidad y los procesos de la PE se desenvuelvan de manera tal, que sus servicios y campo de acción contribuyan con la transformación y desarrollo de la Nación y de las comunidades educativas, ya que con base en atribuciones, se transmite la enseñanza, la investigación, la innovación y la práctica profesional docente, procurando cumplir con uno de los fines de la educación superior, que es transmitir la enseñanza en el contexto de la Escuela, mediante las Prácticas Educativas $^{49}$. Sin olvidar que esta Ley se direccionó desde la misma reforma que sufrió el estado argentino en esta época, donde se aplicó la descentralización estatal, la privatización del espacio público y la desregulación de los servicios públicos $^{50}$.

La Ley de Educación Nacional (26.206/2006) ${ }^{51}$, manifiesta que el espíritu de la formación busca trazar un puente de unión entre la investigación, la enseñanza,

43 "Resolución Nro.32/93 del CFCE. Finalidad y funciones de la Formación Docente Continua" (Argentina, 1993) Consejo Federal de Educación (CFCE). http://www.me.gov.ar/consejo/resoluciones/res93/32-93.pdf.

44 Eva Mara Petitti, “Continuidades y rupturas en la Ley de Educación de la provincia de Buenos Aires (1875-1995)”. Rev. hist.edu. latinoam - Vol. 19 No. 28, enero - junio 2017 - ISSN: 0122-7238 - pp. 223 - 244.

45 "Resolución 36/94 CFCE. Red Federal de Formación Docente Continua" (Argentina, 1994). (CFCE). http://www.me.gov.ar/consejo/ resoluciones/res94/36-94.pdf.

46 "Resolución 52/96 CFCE. Criterios para la reorganización del sistema de Formación Docente" (Argentina, 1996). (CFCE). http:// www.me.gov.ar/consejo/resoluciones/res96/52-96.pdf.

47 "Resolución 105/99 CFCE. Principios y criterios generales para la transformación de la educación de jóvenes y adultos" (Argentina, 1999). (CFCE). http://www.me.gov.ar/consejo/resoluciones/res99/105-99.pdf.

48 “Ley de Educación Superior Nro. 24.521. Decreto 268/95" (Argentina, 1995). http://www.me.gov.ar/consejo/cf_leysuperior.html.

49 Marcela Mollis (2014). "Administrar la crisis de la educación pública y evaluar la calidad universitaria en América Latina: dos caras de la misma reforma educativa”, Revista de la Educación Superior XLIII (1) No.169 (2014): 25-45.

50 Eva Mara Petitti, “Continuidades y rupturas en la Ley de Educación de la provincia de Buenos Aires (1875-1995)”. Rev. hist.edu. latinoam - Vol. 19 No. 28, enero - junio 2017 - ISSN: 0122-7238 - pp. 223 - 244.

51 “Ley de Educación Nacional No. 26.206" (Argentina, 2006). http://www.me.gov.ar/doc_pdf/ley_de_educ_nac.pdf. 
la práctica docente y las experiencias educativas, en la formación inicial y en la continuada de los diferentes niveles educativos e institucionales.

En el 2007, después de la reforma de la Educación en la Argentina, desde el Instituto Nacional de Formación Docente (INFD) se propuso al Ministerio de Educación los "Lineamentos Curriculares Nacionales para la Formación Docente Inicial" ${ }^{2}$, estos fueron el marco regulatorio para los proyectos jurisdiccionales y las prácticas de formación docente inicial en los distintos niveles y modalidades del Sistema Educativo Argentino.

En el documento se destaca la Escuela como Campo de Conocimiento y de escenario de la actuación docente, mediante la participación e incorporación progresiva, del practicante-profesor, en los contextos educativos, con el fin de integrar los otros conocimientos (general y específico), pero dándole énfasis a la formación disciplinar y profesional para el futuro ejercicio docente en distintas áreas, con base en los objetivos, contextos, actividades de campo, prácticas docentes disciplinares, proyectos pedagógicos y comunitarios, capacitación de los profesores-titulares en la PE y desarrollo de procesos evaluativos, hasta abordar plenamente la Residencia Pedagógica Íntegra.

Lo propuesto en el párrafo anterior, se hizo en un proceso que articula e integra proyectos de otros campos del conocimiento, a partir del análisis, experimentación y reflexión en la práctica, ya que la Escuela es considerada como el escenario más apropiado para el desenvolvimiento de la docencia, o sea, se reconoce la importancia de esta o de la institución en general, en el trascurso de la formación docente profesional ${ }^{53}$.

En la misma perspectiva legislativa de la formación docente en Argentina, la Resolución 167/2012 ${ }^{54}$ del Consejo Federal de Educación (C.F.E.), presenta el "Plan Nacional de Formación Docente 2012-2015", propuesto al Ministerio de Educación Nacional, para fortalecer las áreas del Desarrollo Curricular de la Investigación y de la Formación Continuada, por la integración y el apoyo diferenciado en la formación de profesores en la educación superior y en las Escuelas, destacándose como escenario principal el campo de las Prácticas Pedagógicas Educativas, con la articulación administrativa, educativa y pedagógica entre los programas, las instituciones formadoras y los espacios del ejercicio docente.

En la Universidad Nacional de La Plata se encuentra, específicamente para la PE, el documento "Memoria Académica"55, en donde es reglamentada y planteadas las directrices para el desenvolvimiento de la PPE tales como: la relación y determinación de las horas, los objetivos, contenidos, funciones,

52 "Resolución CFE No30/07. Lineamientos Nacionales para la Formación Docente Continua y el Desarrollo Profesional" (Argentina, 2007). Instituto Nacional de Formación Docente (INFD). http://www.me.gov.ar/consejo/resoluciones/res07/30-07.pdf.

53 "Desarrollo Profesional Docente Centrado en la Escuela. Documento Preliminar" (Argentina, 2010). http://es.scribd.com/ doc/48905634/Desarrollo-Profesional-Docente-centrado-en-la-escuela-segunda-parte.

54 “Plan Nacional de Formación Docente 2012-2015. Resolución CFE N¹67/12" (Argentina,2012). Ministerio de Educación, Ciencia y Tecnología. (CFCE). (INFD). http://www.me.gov.ar/consejo/resoluciones/res12/167-12.pdf.

55 "Memoria Académica. Profesorado y Licenciatura en Educación Física. Plan de Estudios 2000". Facultad de Humanidades y Ciencias de la Educación - FaHCE - (Argentina, 2000). Universidad Nacional de La Plata UNLP. La Plata: BIBHUMA. 
requisitos para la aprobación de la PE, modalidades de evaluación, disciplinas que la subsidian, entre otras; además del cómo se promueven y aprueban las Didácticas y Prácticas de Enseñanza; y en el numeral 26 se habla sobre las condiciones para cursar o inscribir la disciplina como Práctica Pedagógica Educativa.

Con base en las diferentes directrices presentadas, se entiende que esos procesos de formación docente, en relación a la PE, son orientadas para el fortalecimiento de la profesión, pero valorizando las realidades educativas a través de la formación en la crítica, en la autonomía, en la creatividad y en el compromiso docente, investigador y comunitario, y en articulación con la reflexión de los conocimientos y experiencias adquiridas, con las directrices gubernamentales e institucionales.

\section{Universidad Metropolitana de Ciencias de la Educación - UMCE - Chile}

El programa de formación de docentes abordado en la investigación fue la Licenciatura en Educación y Pedagogía en Educación Física, Deportes y Recreación; el nombre asignado a la disciplina PE es Práctica Profesional Final.

Los procesos educativos en Chile tuvieron su desarrollo e influencia a partir de documentos como la nueva Constitución de 1980, “Decretos con Fuerza de Ley (DFL)" y la Ley Orgánica Constitucional de Educación (LOCE) de 1990, en que se abordan los principios, los conceptos fundamentales y las disposiciones de carácter administrativo, organizacional y de gestión relativos a la educación, además de la reestructuración de esta en los diferentes niveles de formación ${ }^{56}$.

Ya con base en la nueva dinámica democrática, después del régimen de la dictadura, es presentada la Ley Orgánica Constitucional de Enseñanza (LOCE), $18.962 / 1990^{57}$, la cual ha operado como norma-madre, para la estructuración y funcionamiento del nuevo sistema educativo chileno en todos los niveles. En 1997, fue iniciado el Programa de Fortalecimiento de la Formación Inicial Docente - PFFID -, con el fin de mejorar la calidad del ejercicio docente, a partir de la relación de las políticas y objetivos educativos.

Luego la Ley 20.370/2009 estableció las directrices de una nueva “Ley General de Educación" ${ }^{\prime 58}$ en Chile, en la que se proponen los principios y fines de la educación en general y en la que cada nivel de formación educativa tiene cierta autonomía y libertad para el desarrollo de los procesos de formación profesional.

Sin embargo, la Vice-rectoría Académica de la UMCE, fue más allá de la ley e hizo una modificación en el Reglamento General de Estudios ${ }^{59}$ y aprobó ítems en los que, por ejemplo, para un estudiante de licenciatura obtener su graduación deberá

56 “Informe Nacional de Chile. La Educación Chilena en el Cambio de Siglo: Políticas, Resultados y desafíos" (Chile, 2004) Ministerio de Educación. UNESCO. Oficina Internacional de Educación.

57 “Ley Orgánica Constitucional de Enseñanza ( ${ }^{\circ}$ 18.962)". (Chile, 1990). Ministerio de Educación. http://www.uchile.cl/portal/ presentacion/normativa-y-reglamentos/8386/ley-organica-constitucional-de-ensenanza.

58 "Ley General de Educación. Norma Ley 20370" (Chile, 2009). Ministerio de Educación. https://www.leychile.cl/ Navegar?idNorma=1006043.

59 "Reglamento General de Estudio. Resolución Exenta N698, del 28 de abril de 1992" (Santiago de Chile, 1992). Vicerrectoría Académica. Dirección de Investigación. Universidad Metropolitana de Ciencias de la Educación (UMCE). 
ser competente en todos los requisitos curriculares: y para tal, es obligatoria la culminación de la Práctica Profesional Final (PE), la Memoria o Seminario de Título (Histórico Escolar) y la Prueba escrita de Título.

Esta titulación fue reglamentada por los diferentes programas de formación docente de la Universidad, por eso la UMCE, con base en el artículo 3 de los principios, asumió esencialmente la autonomía para definir sus intereses educativos y la flexibilidad para adaptarse a las realidades, a los proyectos y a las normas vigentes ${ }^{60}$.

Los documentos exhibidos permiten entender la importancia de las directrices y propuestas llevadas a cabo en las reformas curriculares en Chile, estas fueron asumidas por el Reglamento de la PE de la UMCE, en las que la Dirección de la Docencia y la Coordinación General de Prácticas ${ }^{61}$ proporcionan los diferentes direccionamientos para el proceso de la PE. Este reglamento tiene su estructuración basada en el concepto, en los propósitos y en los requisitos para el desarrollo de la PE; en la asistencia y en la responsabilidad de los practicantes-profesores; en la aprobación de la PE; en su proceso, en su organización y administración; en la supervisión; en los requisitos y funciones del profesor-guía (profesor-titular); en la evaluación de la PE y en las postergaciones y homologaciones de la misma.

Además de eso, la constitución del profesional tiene como características una actualización en la formación docente, aplicada y pedagógica, en concordancia con proyectos de las prácticas, de la extensión, de la investigación y de la docencia para el desenvolvimiento de competencias genéricas y específicas que permitan intervenir en los contextos educativos y sociales del área. Por lo que se observa que se constituye un perfil profesional para ser desarrollado en diferentes campos de la Educación Física: promoción de la salud, actividad física, naturaleza y aprovechamiento del tiempo libre y la gestión y organización del entrenamiento físico y deportivo ${ }^{62}$.

Con base en los documentos anteriores y en las directrices presentadas en la misión, en la visión, en los planos de formación y proyectos de desarrollo institucional para la docencia, investigación y extensión, se verifica que se busca promover la profesionalización docente en diversos ámbitos y que, finalmente, la Vice-rectoría Académica de la UMCE, en el Estatuto General de Estudios ${ }^{63}$ de la Universidad definió, que la Orientación Pedagógica que envuelve todos los programas de formación en la Universidad es el Curricular por Competencias.

\section{Reflexiones Relacionales}

Según lo encontrado y relacionado con lo que plantea Rama (2015), todo el proceso de la PE desde la normatividad busca formar profesionales docentes

60 "Reglamento General de Estudio. Resolución 02329 de 2002. Modificación Reglamento General de Estudio". (Chile, 2002). Rectoría. UMCE.

61 "Reglamento de Práctica Profesional Final" (Chile, 2011). Dirección de Docencia. Coordinación General de Prácticas (UMCE).

62 "Licenciatura en Educación y Pedagogía en Educación Física, Deportes y Recreación (Varones)" (Chile, 2015). UMCE. Página Institucional. $\quad$ http://pregrado.umce.cl/index.php/carreras/29-licenciatura-en-educacion-y-pedagogia-en-educacion-fisicadeportes-y-recreacion-varones.

63 "Reglamento General de Estudio. Resolución 02329 de 2002. Modificación Reglamento General de Estudio" (Chile, 2002). Rectoría (UMCE). 
con idoneidad, identidad, critica, análisis y comprensión educativa, pedagógica y didáctica de las problemáticas y realidades de la educación, mediante compromisos con el aprendizaje y la enseñanza, el desarrollo de competencias generales y específicas, y la administración, gestión e investigación educativa

Es evidente que dentro del desarrollo gubernamental de las PE, los procesos se orientan hacia el crecimiento y fortalecimiento de las competencias y habilidades laborales y de praxis, sobresaliendo OP como la Académica y Técnicista, aunque en el contexto universitario se procura por el Construccionismo Social Activo, por medio de la docencia, la investigación y la proyección y la construcción y reconstrucción activa del conocimiento, lo cual evidencia Cuño al plantear que el objeto del Foro de Cooperación Económica Asia-Pacífico (APEC), es que la educación facilite trabajadores para los negocios, la industria y la producción: "Las decisiones sobre educación, - decía el escrito -, deben ser asumidas por el sistema educativo y basadas en razones de negocios y con la máxima intervención posible del sector productivo" 64 .

En los procesos formativos, desde lo legislativo, se evidencia que se valoriza la praxis en estos ${ }^{65}$, predominando en la PE las Orientaciones Pedagógicas Tradicionales, Académicas, de Eficiencia Social, Hermenéuticas y las Centradas en los Procesos

Se devela, según Moon ${ }^{66}$ las relaciones y esferas de un desarrollo personal y profesional con intercambios teóricos, prácticos y realistas en el contexto de la escuela; además de una educación humanizante en torno a lo moral y ético, y una formación para el trabajo dentro del multiculturalismo responsable, profesional y con grandes aportes a la sociedad; ${ }^{67}$ formación compartida entre centros educativos, como lugar de movilización, de producción y de transmisión de las competencias profesionales; ${ }^{68}$ las nuevas Tecnologías, Informáticas Digitales y de Comunicación (TIDC) en los procesos educativos; ${ }^{69}$ la interdisciplinaridad orgánica en la formación de profesores; y la relación, en la Universidad entre la enseñanza, la investigación y la extensión como procesos pedagógicos de formación. ${ }^{70}$

Los programas de formación de docentes, buscan responder a las exigencias de los mercados y de formar para el trabajo bajo el desarrollo de competencias,

64 Justo Cuño Bonito, “La universidad Latinoamericana en la encrucijada: amenazas, desafíos y soluciones”, Rev. hist.edu.latinoam 18, No. 26, (2016), $241-277$.

65 Teresa Cristina Rego, (2012). "Narrativas autobiográficas, singularidades e suas interfaces com a educação: contribuições da perspectiva histórico-cultural" (Tesis de Doctorado en Faculdade de Educação, Universidade de São Paulo, 2012), 404.

66 Robert Moon, "As Políticas transformistas: Transição na formação de professores na Inglaterra", en O Oficio de Professor: Historias perspectivas e desafios internacionais, eds. Maurice Tardif, y Claude Lessard (Petrópolis: Editora Vozes, 2008), 93-111.

67 Pedro Laudionor Goergen y Dermeval Saviani, Formação de Professores: A experiência internacional sob o olhar brasileiro (São Paulo: Autores Associados. NUPES, 1998), 312.

68 Vincente Lang, "Profissão de Professor na França: Permanência e fragmentação", en O Oficio de Professor: Historias perspectivas e desafios internacionais, eds. Maurice Tardif, y Claude Lessard (Petrópolis: Editora Vozes, 2008), 136 -152.

69 Pierre Lévy, As tecnologias da inteligência: o futuro do pensamento na era da informática (Rio de Janeiro: Nova Fronteira,1994), 203.

70 Carmen Lucia Freitas de Castro, "Novas Políticas de Formação: Da concepção negada à concepção consentida", en Trajetórias $e$ perspectivas da formação de educadores, ed. Raquel Lazzari Leite Barbosa. (São Paulo: Editora UNESP, 2004), 89-116. 
la apropiación de lo académico de estos mismos procesos, en que el espacio de la PE sea punto de conexiones y de encuentros entre universidad y escuela. ${ }^{71}$

Prevalece el espíritu de OP Contemporáneas, en los documentos, no obstante en su ejecución es evidente que sobresalen las OP Conservadoras como la Técnicista centrada en la Eficacia Social y en la Tradicional o Teórica, todo esto centrado en una lógica de transmitir-adquirir conocimientos, de lo financiero y de una economía sostenida, fortaleciendo la masificación y diversificación formativa de maestros, o sea, preparar mano de obra competente para la "mercadotecnia" y la "ideología del mercado"72.

\section{CONCLUSIÓN}

En las cuatro Universidades objeto de pesquisa y sus respectivos países, se comprende que la Práctica Educativa e incluso los procesos de formación de docentes para la Educación Física, el desarrollo de estas tiene como base principal responder desde las construcciones teóricas que se encuentran en las normatividades (leyes, legislaciones, reglamentaciones y directrices del gobierno), encontrando que las Orientaciones Pedagógicas se desenvuelven entre las Contemporáneas y Conservadoras, aunque es evidente que a nivel gubernamental, lo presentado, busca responder a las exigencias de lo financiero que determinan las inversiones en la educación, esencialmente, fomentando una educación de masas para lo laboral y el desarrollo industrial, como acomodación a las exigencias económicas, financieras y laborales de las multinacionales y del capitalismo en sí, o sea "capital humano especializado"73.

Lo anterior, convoca a que los investigadores y actores educacionales profundicemos, en lo que es el espíritu y la propiedad que hay en las normas que direccionan los desarrollos de la educación en la América Latina, para comprender el verdadero fin de estas, desde las instancias gubernamentales y asumir los roles respectivos y adecuados a las necesidades de los contextos. .

Al analizar los resultados, desde las perspectivas universitarias y específicamente dentro de los procesos de formación docente, las Orientaciones Pedagógicas del cuño Contemporáneo como la Centrada en los Procesos y la Hermenéutica (Critica Reflexiva) son las que esencialmente predominan en los documentos y en el desarrollo de los procesos, entendiendo que el objetivo es formar profesionales docentes reflexivos, con capacidad adaptativa a la enseñanza y al aprendizaje real y contextual educativo; que posea habilidades,

71 Licínio Lima, Mário Luiz Neves de Azevedo y Afrânio Mendes Catani, "O processo de Bolonha, a avaliação da educação superior e algumas considerações sobre a Universidade Nova”, Avaliação13, No 1 (2008), 7-36. http://dx.doi.org/10.1590/S141440772008000100002.

72 Marcela Mollis, "Administrar la crisis de la educación pública y evaluar la calidad universitaria en América Latina: dos caras de la misma reforma educativa”. Revista de la Educación Superior XLIII (1) No. 169, (2014), 25-45.

73 Justo Cuño Bonito, "La universidad Latinoamericana en la encrucijada: amenazas, desafíos y soluciones", Rev. hist.edu.latinoam 18, No. 26, (2016), 241 - 277. 
capacidades y competencias pedagógicas adquiridas desde las experiencias, los conocimientos y en el compartir con los pares (estudiantes y profesores) hacia la construcción de su identidad docente.

Como encerramiento provisorio, entendemos que el desarrollo profesional de los profesores y la formación docente de los alumnos, se desenvuelven en torno a directrices y normas producto de lo gubernamental, las cuales tienen fuerte incidencia en todo lo que implica en las historias de vida de los actores y de sus contextos como actores critico-sociales y políticos.

Agradecimientos: A la Asociación de Universidades Iberoamericanas y Posgrados (AUIP), Universidad Estadual Paulista (UNESP) Campus Río Claro (SP), Universidad de Caldas (Manizales, Colombia).

\section{FUENTES}

Decreto No. 87.497. Agosto 18 de 1982. Regulamenta a Lei No. 6.494, de 07 de dezembro de 1977, Regulamenta a Lei No. 6.494, de 07 de dezembro de 1977. Brasília. D.F. - Brasil. Presidência da República. http://www.planalto.gov.br/ccivil_03/decreto/ d87497.htm

Ley de Educación Superior No. 24.521. Decreto 268/95. Buenos Aires - Argentina. http:// www.me.gov.ar/consejo/cf_leysuperior.html.

Ley de Educación Nacional No. 26.206/06. Buenos Aires - Argentina (MEN). http:// www.me.gov.ar/doc_pdf/ley_de_educ_nac.pdf.

Ley General de Educación. Ley 115 de 1994. Promulgada febrero 08 de 1994. Bogotá. D.C. - Colombia. http://www.mineducacion.gov.co/1621/articles-85906_archivo_ pdf.pdf.

Ley General de Educación. Norma Ley 20370/09. Santiago de Chile - Chile. Ministerio de Educación. https://www.leychile.cl/Navegar?idNorma=1006043.

Ley Orgánica Constitucional de Enseñanza ( $\mathrm{N}^{\circ}$ 18.962). Santiago de Chile - Chile. Ministerio de Educación. http://www.uchile.cl/portal/presentacion/normativa-yreglamentos/8386/ley-organica-constitucional-de-ensenanza.

Reglamento de Práctica e Investigación Educativa de los Programas de Licenciatura de la Universidad de Caldas, Manizales - Colombia. Universidad de Caldas (UdeC).

Facultad de Artes y Humanidades, Departamento de Estudios Educativos.

Reglamento General de Estudio. Resolución Exenta N698, del 28 de abril de 1992. Santiago de Chile - Chile. Vicerrectoría Académica. Dirección de Investigación. Universidad Metropolitana de Ciencias de la Educación (UMCE).

Reglamento General de Estudio. Resolución 02329 de 2002. Modificación Reglamento General de Estudio. Santiago de Chile - Chile. Rectoría. UMCE.

Reglamento de Práctica Profesional Final. Santiago de Chile - Chile. Dirección de Docencia. Coordinación General de Prácticas (UMCE).

Resolução 36 de 7 de agosto de 1996. Obrigatoriedade do Estágio Curricular Obrigatório. Rio Claro (SP) - Brasil. Reitoria. Universidade Estadual Paulista (UNESP).

Resolução CNE/CP 1/2002. Institui Diretrizes Curriculares Nacionais para a Formação de Professores da Educação Básica, em nível superior, curso de licenciatura, de 
graduação plena. Brasília D.F. - Brasil. http://portal.mec.gov.br/cne/arquivos/pdf/ rcp01_02.pdf.

Resolução CNE/CP 2, de 19 de fevereiro de 2002. Institui a duração e a carga horária dos cursos de licenciatura, de graduação plena, de formação de professores da Educação Básica em nível superior" D. O. U. Brasília D.F. - Brasil. Ministério da Educação.

Resolução N 7, Março 31 de 2004. Institui as Diretrizes Curriculares Nacionais para os cursos de graduação em Educação Física, em nível superior de graduação plena. Brasília D.F. - Brasil. Câmara de Educação Superior. http://portal.mec.gov.br/cne/ arquivos/pdf/ces0704edfisica.pdf.

Resolución Nro.32/93 del CFCE. Finalidad y funciones de la Formación Docente Continua. Buenos Aires - Argentina. Consejo Federal de Educación (CFCE). http://www.me.gov.ar/consejo/resoluciones/res93/32-93.pdf.

Resolución 36/94 CFCE. Red Federal de Formación Docente Continua. Buenos Aires Argentina. (CFCE). http://www.me.gov.ar/consejo/resoluciones/res94/36-94.pdf.

Resolución CFE №30/07. Lineamientos Nacionales para la Formación Docente Continua y el Desarrollo Profesional. Buenos Aires - Argentina. Instituto Nacional de Formación Docente (INFD). http://www.me.gov.ar/consejo/resoluciones/ res07/30-07.pdf.

Resolución 5443 de 2010. Promulgada junio 30 de 2010. Ministerio de Educación Nacional (MEN). Bogotá D.C. - Colombia. www.mineducacion.gov.co/.../articles-243532_ archivo_pdf_res6966.pdf.

Resolución 52/96 CFCE. Criterios para la reorganización del sistema de Formación Docente. Buenos Aires - Argentina. (CFCE). http://www.me.gov.ar/consejo/ resoluciones/res96/52-96.pdf.

Resolución 105/99 CFCE. Principios y criterios generales para la transformación de la educación de jóvenes y adultos. Buenos Aires - Argentina. (CFCE). http://www. me.gov.ar/consejo/resoluciones/res99/105-99.pdf.

\section{REFERENCIAS}

Andrade Oliveira, Dalila. "Reformas educativas y redefinición de las fronteras entre lo público y lo privado", en Políticas de privatización, espacio público y educación en América Latina, editado por Pablo Gentili, Gaudêncio Frigotto, Roberto Leher y Florencia Stubrin. Santa Fe: Homo Sapiens Ediciones, 2009, 121-134.

Ayala Zuluaga, Carlos Federico, Franco Jiménez, Alejandra María y Ayala Zuluaga, José Enver. "Prácticas profesionales educativas (PPE): reflexión desde la educación física", Revista Latinoamericana de Ciencias Sociales, Niñez y Juventud, 13, no. 2 (2015): 595-607. DOI: http://dx.doi.org/10.11600/1692715x.1323100713.

Bauer, Carlos y Paiva, Luis. O Sindicalismo docente universitário da Argentina, Brasil, Colômbia, México e sua resistência à contrarreforma universitária. Rev. hist. edu.latinoam, 19 no. 28, (2017): 109 - 128. ISSN: 0122-7238 - DOI: https://doi. org/10.19053/01227238.5210. 
Carvalho Pessoa de, Anna Maria. Prática de Ensino: os estágios na formação do professor. São Paulo: Biblioteca Pioneira de Ciências Sociais, 1985, 106.

Cuño Bonito, Justo. La universidad Latinoamericana en la encrucijada: amenazas, desafíos y soluciones, Rev. hist.edu.latinoam 18, No. 26, (2016): 241 - 277. ISSN: 0122-7238. DOI: https://doi.org/10.19053/01227238.4374.

Desarrollo Profesional Docente Centrado en la Escuela. Documento Preliminar. Buenos Aires - Argentina. http://es.scribd.com/doc/48905634/Desarrollo-ProfesionalDocente-centrado-en-la-escuela-segunda-parte.

Faria Junior Gomes de, Alfredo, Corrêa da Silva, Eugénio y Bressane da Silva, Riselaine. Prática de ensino em educação física: estágio supervisionado. Rio de Janeiro: Interamericana, 1982, 285.

Feiman-Nemser, Sharon. "Teacher Preparation: Structural and conceptual alternatives", en Handbook of research on teacher education editado por Robert Houston. New York: Mac Millan, 1990, 212-233.

Ferreira Bartolozzi, Eliza. “Ensino Meio no Brasil: Os desafios das políticas de garantia do direito a sua universalização". Linhas Críticas, Revista da Faculdade de Educação - UnB 17, No. 34 (2011): 507-525.

Freidson, Eliot. Renascimento do profissionalismo: teoria, profecia e política. São Paulo: Editora da Universidade de São Paulo, 1998, 280.

Freitas de Castro, Carmen Lucia. “Novas Políticas de Formação: Da concepção negada à concepção consentida", en Trajetórias e perspectivas da formação de educadores, editado por Raquel Lazzari Leite Barbosa. São Paulo: Editora UNESP, 2004, 89116.

Goergen, Pedro Laudionor y Saviani, Dermeval. Formação de Professores: A experiência internacional sob o olhar brasileiro. São Paulo: Autores Associados. NUPES, 1998, 312.

Imbernón, Francisco. La Formación y el Desarrollo Profesional del Profesorado: Hacia una nueva cultura profesional. Barcelona: Editorial Grau, 2007.

Informe Nacional de Chile. La Educación Chilena en el Cambio de Siglo: Políticas, Resultados y desafíos. Santiago de Chile - Chile. Ministerio de Educación. UNESCO. Oficina Internacional de Educación.

Lang, Vincente. "Profissão de Professor na França: Permanência e fragmentação", en O Oficio de Professor: Historias perspectivas e desafios internacionais, editado por Maurice Tardif, y Claude Lessard. Petrópolis: Editora Vozes, 2008, 136 -152.

Lei No 6.494, de 7 de dezembro de 1977. Dispõe sobre o estágio de estudante de estabelecimentos de ensino superior e de $2^{\circ}$ Grau Regular e Supletivo, nos limites que especifica e dá outras providências. Brasília D.F. - Brasil. Casa Civil. Subchefia para Assuntos Jurídicos. http://www.planalto.gov.br/ccivil_03/leis/L6494.htm.

Lei no̊8.859. Março 23 de 1994. D. O. U de 24/03/94. Estagiários - Aceitação pelas pessoas jurídicas de Direito Privado - Órgãos da Administração Pública e Instituições de Ensino - Requisitos - Critérios - Lei no 6.494/77 Alteração. Brasília D.F. - Brasil. Congresso Nacional. http://www4.pr.gov.br/gee/documentos/legislacao/LEI8859-94-ESTAGIO.pdf.

Lei n. 11.788 setembro 25 de 2008. Disposições sobre Estágio de Estudantes. Brasília D.F. - Brasil. Presidência da República. http://www.planalto.gov.br/ccivil_03/_ ato2007-2010/2008/lei/111788.htm. 
Lévy, Pierre. As tecnologias da inteligência: o futuro do pensamento na era da informática. Rio de Janeiro: Nova Fronteira,1994, 203.

Licenciatura en Educación y Pedagogía en Educación Física, Deportes y Recreación (Varones). Santiago de Chile - Chile. UMCE. Página Institucional. http://pregrado. umce.cl/index.php/carreras/29-licenciatura-en-educacion-y-pedagogia-eneducacion-fisica-deportes-y-recreacion-varones.

Lima Licínio, Mário, Azevedo Neves de, Luiz y Catani Mendes, Afrânio. “O processo de Bolonha, a avaliação da educação superior e algumas considerações sobre a Universidade Nova", Avaliação 13, no 1 (2008): 7-36. http://dx.doi.org/10.1590/ S1414-40772008000100002.

Lima Lucena, Maria Socorro y Pimenta Garrido, Selma. Estágio e Docência. São Paulo: Cortez Editora, 2004.

Loya Chávez, Hermila. "Los Modelos Pedagógicos en la Formación de Profesores", Revista Ibero-americana de Educación. Organización de Estados Ibero-americanos para la Educación, la Ciencia y la Cultura (OEI). (2008): sp.

Melo Ferreira, Geovana. "Estágio na Formação Inicial de Professores: Aguçando o olhar, desenvolvendo a escuta sensível", en Estágio Supervisionado e Prática de Ensino: Desafios e possibilidades, editado por Lázara Cristina da Silva y Maria Irene Miranda. Araraquara: Junqueira\&Marin Editores, 2008, 85-113.

Memoria Académica. Profesorado y Licenciatura en Educación Física. Plan de Estudios 2000. La Plata (BA) - Argentina. Facultad de Humanidades y Ciencias de la Educación - FaHCE - Universidad Nacional de La Plata UNLP. La Plata: BIBHUMA.

Milanesi, Irton. O estágio interdisciplinar no processo de formação docente. Cáceres: UNEMAT Editora, 2008, 198.

Mollis, Marcela (2014). “Administrar la crisis de la educación pública y evaluar la calidad universitaria en América Latina: dos caras de la misma reforma educativa". Revista de la Educación Superior XLIII (1) No.169 (2014): 25-45.

Moon, Robert. "As Políticas transformistas: Transição na formação de professores na Inglaterra", en O Oficio de Professor: Historias perspectivas e desafios internacionais, editado por. Maurice Tardif, y Claude Lessard. Petrópolis: Editora Vozes, 2008, 93-111.

Parecer N..: CNE/CP 27/2001. Dá nova redação ao item 3.6, a línea c, do Parecer CNE/CP 9/2001, que dispõe sobre as Diretrizes Curriculares Nacionais para a Formação de Professores da Educação Básica, em nível superior, curso de licenciatura, de graduação plena. Brasília D.F. - Brasil. Consejo Nacional de Educación (CNE). http://portal.mec.gov.br/cne/arquivos/pdf/027.pdf.

Parecer N.o: CNE/CP 28/2001. Dá nova redação ao Parecer CNE/CP 21/2001, que estabelece a duração e a carga horária dos cursos de Formação de Professores da Educação Básica, em nível superior, curso de licenciatura, de graduação plena. Brasília D.F. - Brasil. http://portal.mec.gov.br/cne/arquivos/pdf/028.pdf.

Parecer CNE/CES №: 416/2012: Consulta sobre estágio no exterior. Brasília D.F. - Brasil. http://www.prograd.ufu.br/legislacoes/parecer-cneces-no-4162012-aprovadoem-8-de-novembro-de-2012-estagio-no-exterior. 
Petitti, Eva Mara. “Continuidades y rupturas en la Ley de Educación de la provincia de Buenos Aires (1875-1995)". Rev. hist.edu.latinoam, 19 No. 28 (2017): 223 - 244. ISSN: 0122-7238. DOI: https://doi.org/10.19053/01227238.5546.

Plan Nacional de Formación Docente 2012-2015. Resolución CFE N¹67/12. Buenos Aires - Argentina. Ministerio de Educación, Ciencia y Tecnología. (CFCE). (INFD). http://www.me.gov.ar/consejo/resoluciones/res12/167-12.pdf.

Perrotta, Daniela. “Organismos Internacionales y Políticas en Educación Superior: su aporte al campo de estudios de la educación superior y a la construcción de políticas públicas". Revista de la Educación Superior. XLIV (1); No. 173 (2015): 181188.

Política Curricular. Programa de Licenciatura en Educación Básica con Énfasis en Educación Física, Recreación y Deporte, Manizales - Colombia. Universidad de Caldas (UdeC). Consejo Académico: 14.

Proposta de reestruturação curricular dos cursos de licenciatura e graduação (bacharelado) em educação física do instituto de biociências da UNESP/RC. Rio Claro (SP) - Brasil. Conselho de cursos de graduação em educação física - CCGEF Comissão de estudo curricular - CEC. UNESP.

Rama, Claudio. “La conformación diferenciada de un nuevo subsistema tecnológico universitario en América Latina". Revista de la Educación Superior. XLIV (1), No. 173 (2015): 11-46.

Rego, Teresa Cristina. "Narrativas autobiográficas, singularidades e suas interfaces com a educação: contribuições da perspectiva histórico-cultural". (Tesis de Doctorado en, Faculdade de Educação, Universidade de São Paulo, 2012, 404.

Ruiz Larraguivel. Estela. "Los técnicos superiores universitarios. Diferenciación educativa, estratificación social y segmentación del trabajo". Revista Mexicana de Sociología, 71, No. 3, (2009): 557-584.

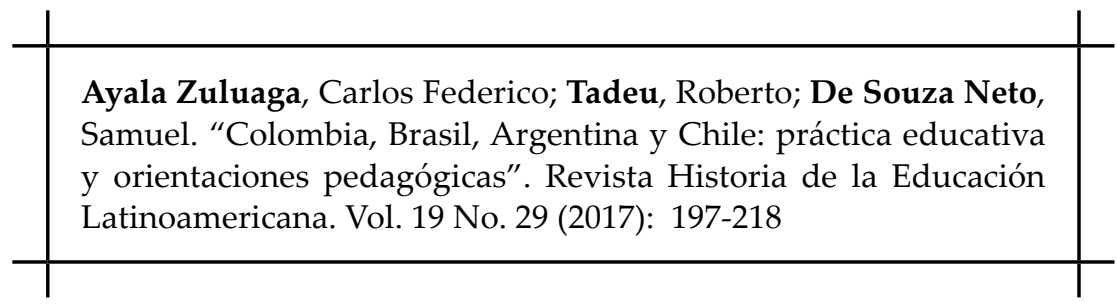

